\title{
Effects of brown rice powdered drink on glucose control and lipid profile of type 2 diabetes patients.
}

\author{
Husna Mansor ${ }^{1}$, Lili Husniati Yaacob ${ }^{*}$, Azidah Abdul Kadir ${ }^{1}$, Wan Mohd Zahiruddin Wan \\ Mohammad $^{2}$
}

${ }^{1}$ Department of Family Medicine, Universiti Sains Malaysia, Kota Bharu, Kelantan, Malaysia

${ }^{2}$ Department of Community Medicine, Universiti Sains Malaysia, Kota Bharu, Kelantan, Malaysia

\begin{abstract}
Diabetes mellitus causes multiple adverse health outcomes. Thus, control of the disease is paramount in preventing its complications. Brown rice, as a whole-grain staple, has been proven to be beneficial in many of these respects. However, due to taste and cost, many consumers avoid brown rice as an alternative to white rice. Our primary aim was to study the effects of a brown rice instant drink on glycemic control, lipid profile, weight, and blood pressure in patients with uncontrolled diabetes. This was a randomized, open-label clinical trial conducted over 12 w. Sixty-four participants were randomized into an intervention group and a control group. The intervention group received $18 \mathrm{~g}$ of powdered brown rice to be consumed in a drink three times daily for $12 \mathrm{w}$. The control group was given standard or their usual treatment. Fasting plasma glucose, HbA1c, plasma lipid, blood pressure, and weight were measured and recorded before and after the intervention. There were no significant differences between the two groups in terms of HBA1c levels at the end of the intervention $(P=0.081)$. However, the BR group had a reduction in HbA1c with a mean of $0.11 \%$, and the control group had an increase of HbA1c of $0.25 \%$. There was significant improvement in total cholesterol, triglycerides, and blood pressure levels at the end of the intervention $(\mathbf{P}<0.005)$ in the intervention group. In conclusion, brown rice instant drink has improved total cholesterol, triglycerides, and systolic and diastolic blood pressure in subjects with type 2 diabetes.
\end{abstract}

Keywords: Brown rice, Glucose control, Lipid, Diabetes mellitus.

Accepted on December 11, 2018

\section{Introduction}

Globally, type 2 diabetes is on the rise. The World Health Organization (WHO) reported that in 2014, the global prevalence of diabetes was $8.5 \%$. This is equal to 422 million adults, nearly $50 \%$ of whom are estimated to live in Southeast Asia and other Western Pacific regions [1]. This figure has increased from $4.7 \%$ in 1980 [2]. The leading risk factors responsible for this dramatic rise are obesity, urbanization, and sedentary lifestyles [3].

The highest rates of uncontrolled diabetes occur in the Southeast Asian, Eastern Mediterranean, and African regions [1]. Multiple, diverse approaches, including lifestyle changes and pharmacological treatments, are needed to ensure effective glycemic control of diabetes. Medical nutritional therapy and dietary modification may help glycemic control in the long term [4-6]. Effective dietary modification, for example, has been shown to aid in reducing $\mathrm{HbAlc}$ by an average of $1-2 \%$ [3]. It has also been shown to help improve other metabolic indices, such as cholesterol levels, when added as an adjunct to medical therapy [6].
One of the healthier food choices that is increasingly being studied is brown rice (Oryza sativa). Brown rice contains many micronutrients in its bran. As a whole, increased fiber and micronutrients in daily staple foods could help improve triglycerides and total cholesterol levels, as shown in a previous meta-analysis [7]. Brown rice in the form of germinated brown rice has also been found to improve glycemic control in pre-diabetic people [8]. Other metabolic indices, such as weight and blood pressure levels, are also improved by substituting white rice with brown rice in the daily diet [9].

Brown rice has previously been shown to be better than white rice in terms of health benefits. Moreover, many previous studies have shown that consuming white rice in the long term could be a risk factor for developing type 2 diabetes [10,11]. This is mainly due to the high glycemic index and high glucose load of white rice $[12,13]$.

Despite the proven benefits of brown rice, not many people have included it in their daily staple. This is likely due to its taste and cost. Therefore, attempts to promote the benefits of brown rice among consumers, in beverages and other food 
products, have been developed. None of the previous studies involving brown rice have studied its benefit as a supplement or in its powdered form. Therefore, in this study, we aimed to determine the overall effects of instant brown rice powder as an adjunct to concurrent medical therapy for the glycemic control of type 2 diabetic patients, as well as its effects on other related metabolic indices, namely lipid control, blood pressure, and weight.

\section{Material and Methods}

Participants were recruited from the outpatient clinic of Hospital Universiti Sains Malaysia Hospital, in Kelantan, Malaysia from July 2016 through November 2016. The inclusion criteria were as follows: ages 18 to 70 years old, diagnosis of type 2 diabetes mellitus for at least six months, HbAlc level between $6.5 \%$ and $9 \%$ within the last three months, use of oral hypoglycemic medication, and no ingestion of any other type of supplement or traditional herbal medication throughout the study. Patients with end-stage renal disease or proliferative retinopathy due to diabetes, as well as pregnant women and patients taking insulin, were excluded from the study.

Withdrawal criteria included any major changes in the participants' concurrent medication which could have affected the results of the study, the ingestion of any other type of herbal or nutritional supplements at any time during the duration of the study, the occurrence of any intolerable or unacceptable adverse events, and/or if the investigator felt that a participant was unfit to continue the study for reasons unrelated to the product. Thus, at the end of the study, three participants were withdrawn for various reasons. One participant could not be contacted for follow-up two weeks after the randomization. Another participant was withdrawn because she did not consume any of her concurrent medication, which the investigator thought could be detrimental to her health. The other participant requested to be withdrawn from the study as she could not tolerate the taste of the product.

Powdered brown rice drink from the brand, "Nature's Own," was used in the intervention group as the adjunctive therapy product. This powdered brown rice is made of pure brown rice with no additives. Participants were instructed to mix 3 tablespoons of the powder, which is equal to $18 \mathrm{~g}$, into $250 \mathrm{ml}$ of water. They were instructed to take the intervention three times per day about 15 min prior to their next meal.

Participants in the control group were not given any supplement. They were only instructed to continue their medication and usual diet.

\section{Sample size calculation}

Sample size calculation was completed using power and sample size software with a power of study at $80 \%$ and $\alpha$ level of 0.05 . The total number of participants was determined after calculating the sample size for each objective independently. The largest sample size was taken as the study's sample size: 64 participants. This figure included a 20-percent drop-out rate.
The sample size calculation was based on the HbA1c variable with a significant difference of $0.18 \%$ and a standard deviation of 0.23 , taken from a study conducted by Hayakawa et al. in 2009 [14].

\section{Study design and randomization}

A randomized, open-label clinical trial was conducted without the blinding of the investigational product. All participants were advised not to consume any special diet or meals throughout the whole study. Participants were randomized into two groups: the control group (CG) and the intervention group (BR). A computer-generated randomization list using a block of 4 was used for the randomization process. The allocated sequence was concealed from the researcher enrolling and assessing the participants. The participants were sequentially numbered and the data were placed in opaque, sealed, and stapled envelopes.

\section{Study procedure}

Upon their consent, participants' basic information and demographic data were collected, including current morbidities and medications. Blood was then drawn to determine baseline biochemical levels of fasting plasma glucose, fasting lipid profile, and HbAlc levels. Physical examination of the participants was performed to measure their blood pressure, height, and weight.

Those in the CG group were given general advice on compliance with medication requirements and an update on their baseline results. They were seen again after $12 \mathrm{w}$ for a final meeting, blood withdrawal for post-intervention biochemical evaluation, and a physical examination, as well as to confirm concurrent medications being taken.

Participants in the BR group were given the instant brown rice powder and specific instructions for its preparation. They were seen after two weeks to ensure compliance and to assess any side effects of the product. They were then given more brown rice powder for the remaining $10 \mathrm{w}$ of the study period. The final follow-up took place after $12 \mathrm{w}$ of product consumption to collect final data on biophysical parameters, product compliance, and confirmation of concurrent medications being taken (Figure 1).

Any side effects of the product were evaluated as well. Blood was also drawn for biochemical evaluation of the study postintervention. Compliance was determined by weighing the residual product remaining within the participants, which was calculated against the total amount of product given.

The study protocol was reviewed and given clearance by the Institutional Review Board of the Research Ethics Committee (Human), Universiti Sains Malaysia (IRB No.: IRB00004494). This research was registered with the Thai Clinical Trials Registry (TCTR) with identification number TCTR20170608002. 


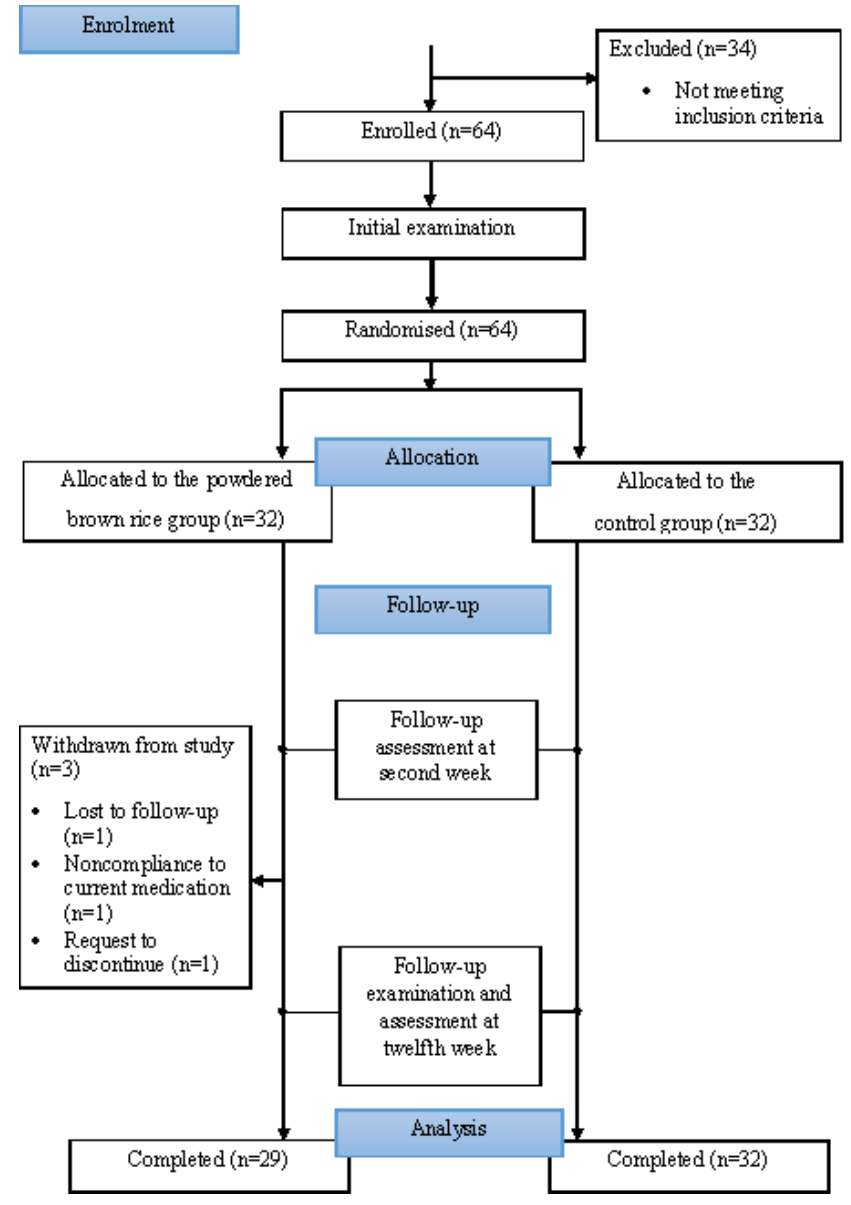

Figure 1. Flow chart of the study.

\section{Statistical analysis}

Statistical analysis was conducted using SPSS software version 22 (SPSS Inc., USA). Baseline characteristics and clinical data were calculated using descriptive statistical analysis. Normally distributed continuous variables were analysed using independent t-tests to determine differences between means.

The primary endpoint, to determine the effects of instant brown rice drink on glycemic control, specifically HbAlc and fasting plasma glucose, was analysed using RM-ANOVA. The secondary endpoint, which was to assess the effects of the brown rice drink on total cholesterol, HDL cholesterol, LDL cholesterol, triglycerides, weight, body mass index (BMI), systolic blood pressure (SBP), and diastolic blood pressure (DBP), were also calculated using RM-ANOVA. Statistical significance was based on the overall group $\times$ time outcome of each variable.

\section{Results}

\section{Demographics and clinical and biochemical results at baseline}

Results were based on the remaining 61 participants. Of them, the mean age was $56.41 \pm 8.09$. Participants comprised 33 men and 28 women. Baseline HbA1c, FBS, total cholesterol, HDL cholesterol, LDL cholesterol, triglycerides, weight, BMI, SBP, and DBP are shown in Table 1 and were comparable between the control and intervention groups.

Table 2 shows a summary of the results for all participants at baseline and at $12 \mathrm{w}$, as well as the mean difference after intervention with the instant brown rice drink compared to the control group. There was a non-significant trend in favor of the intervention group in the reduction of HbAlc. A mean reduction of $0.11 \%$ was seen in the intervention group. Whereas in the control group, there was an increase in the mean $\mathrm{HbA} 1 \mathrm{c}$ of $0.25 \%$. As for FBS levels, there was a nonsignificant increase in both groups, albeit with a greater increment in the control group.

There was a significant improvement of total cholesterol and triglyceride levels in the $\mathrm{BR}$ group ( $\mathrm{TC}: \mathrm{P}=0.016$; $\mathrm{TG}$ : $\mathrm{P}=0.018)$. LDL cholesterol showed a non-significant trend in favor of the BR group, with the mean of the control group increasing by $0.05 \mathrm{mmol} / \mathrm{L}$ in contrast to the BR group, which improved by $0.23 \mathrm{mmol} / \mathrm{L}$. HDL cholesterol did not show much change in the mean difference in both groups $(\mathrm{P}=0.41)$.

The weight and BMI of both groups showed a non-significant incremental trend (weight: $\mathrm{P}=0.431$; $\mathrm{BMI}$ : p-value=0.401). However, interestingly, SBP and DBP showed a significant reduction in the $\mathrm{BR}$ group for the group $\times$ time interaction (SBP: $\mathrm{P}=0.02$; DBP: $\mathrm{P}=0.001$ ).

Table 1. Baseline biochemical and clinical characteristics of participants between CG and BR groups.

\begin{tabular}{|c|c|c|c|c|c|}
\hline \multirow[t]{2}{*}{ Variables } & \multicolumn{2}{|l|}{ Mean (SD) } & \multirow[t]{2}{*}{ Mean diff $(95 \% \mathrm{Cl})$} & \multirow[t]{2}{*}{ t-statistics (df) } & \multirow[t]{2}{*}{ P-value } \\
\hline & Control & Brown rice & & & \\
\hline HbA1c (\%) & $7.68(0.49)$ & $7.71(0.61)$ & $-0.03(-0.31,0.25)$ & $-0.23(59)$ & 0.818 \\
\hline FBS (mmol/L) & $7.65(1.56)$ & $7.13(2.20)$ & $0.52(-0.45,1.49)$ & $1.08(59)$ & 0.285 \\
\hline $\mathrm{TC}(\mathrm{mmol} / \mathrm{L})$ & $4.73(1.14)$ & $5.07(1.05)$ & $-0.34(-0.91,0.22)$ & $-1.21(59)$ & 0.233 \\
\hline $\mathrm{HDL}(\mathrm{mmol} / \mathrm{L})$ & $1.32(0.24)$ & $1.30(0.28)$ & $0.02(-0.11,0.15)$ & $0.28(59)$ & 0.778 \\
\hline LDL (mmol/L) & $2.75(0.90)$ & $2.99(0.85)$ & $-0.23(-0.68,0.22)$ & $-1.03(59)$ & 0.308 \\
\hline TG (mmol/L) & $1.46(0.49)$ & $1.74(0.98)$ & $-0.28(-0.70,0.13)$ & $-1.37(38.33)$ & 0.179 \\
\hline
\end{tabular}




\begin{tabular}{|c|c|c|c|c|c|}
\hline Weight (kg) & $71.37(13.32)$ & $70.42(11.48)$ & $0.95(-5.48,7.38)$ & $0.30(59)$ & 0.769 \\
\hline BMI (kg/m²) & $27.95(4.85)$ & $26.91(3.36)$ & $1.04(-1.13,3.22)$ & $0.96(59)$ & 0.341 \\
\hline $\mathrm{SBP}(\mathrm{mmHg})$ & $131.24(15.66)$ & $138.50(12.93)$ & $-7.26(-14.70,0.18)$ & $-1.95(59)$ & 0.056 \\
\hline $\mathrm{DBP}(\mathrm{mmHg})$ & $78.42(10.37)$ & 82.39 (7.66) & $-3.97(-8.60,1.66)$ & $-1.72(57.96)$ & 0.092 \\
\hline
\end{tabular}

HbA1c: Glycosylated Hemoglobin; FBS: Fasting Blood Sugar; TC: Total Cholesterol; HDL: High Density Lipoprotein Cholesterol; LDL: Low Density Lipoprotein Cholesterol; TG: Triglycerides; BMI: Body Mass Index; SBP: Systolic Blood Pressure; DBP: Diastolic Blood Pressure; SD: Standard Deviation.

Table 2. Biochemical and clinical outcomes of participants.

\begin{tabular}{|c|c|c|c|c|c|c|c|c|}
\hline \multirow[t]{3}{*}{ Variables } & \multicolumn{3}{|c|}{ Control Group (CG) (n=29) } & \multicolumn{3}{|c|}{ Brown Rice Group (BR) $(n=32)$} & \multirow{2}{*}{$\begin{array}{l}\begin{array}{l}\text { Group } \\
\text { statistics }\end{array} \\
\text { F-stats (df) }\end{array}$} & \multirow{2}{*}{$\begin{array}{l}\text { ie interaction } \\
\text { P-value }\end{array}$} \\
\hline & Mean (SD) & & Mean diff $(95 \% \mathrm{Cl})$ & Mean (SD) & & Mean diff $(95 \%$ & & \\
\hline & Baseline & $12 \mathrm{w}$ & Baseline & $12 w$ & & & & \\
\hline $\mathrm{HbA} 1 \mathrm{c}$ & $7.68(0.49)$ & $7.93(0.89)$ & $0.25(-0.03,0.52)$ & $7.71(0.61)$ & $7.60(0.93)$ & $-0.11(-0.43,0.21)$ & $3.15(1)$ & 0.081 \\
\hline FBS & $7.65(1.56)$ & $8.90(2.04)$ & $1.25(0.62,1.89)$ & $7.13(2.20)$ & $7.99(2.29)$ & $0.86(-0.13,1.85)$ & $0.49(1)$ & 0.486 \\
\hline Total Cholesterol & $4.73(1.14)$ & $4.94(1.15)$ & $0.21(-0.06,0.49)$ & $5.07(1.05)$ & $4.79(0.87)$ & $-0.28(-0.58,0.02)$ & $6.13(1)$ & 0.016 \\
\hline HDL cholesterol & $1.32(0.24)$ & $1.32(0.21)$ & $0.00(-0.05,0.05)$ & $1.30(0.28)$ & $1.26(0.23)$ & $-0.03(-0.10,0.03)$ & $0.689(1)$ & 0.41 \\
\hline LDL cholesterol & $2.75(0.91)$ & $2.80(0.90)$ & $0.05(-0.17,0.27)$ & $2.99(0.85)$ & $2.77(0.70)$ & $-0.21(-0.44,0.01)$ & $2.92(1)$ & 0.093 \\
\hline Triglycerides & $1.46(0.49)$ & $1.81(0.88)$ & $0.35(0.09,0.60)$ & $1.74(0.98)$ & $1.65(0.67)$ & $-0.09(-0.35,0.17)$ & $5.94(1)$ & 0.018 \\
\hline Weight & $71.37(13.32)$ & $72.02(13.47)$ & $0.65(0.18,1.11)$ & $70.42(11.48)$ & $70.63(11.70)$ & $0.20(-0.91,1.32)$ & $0.63(59)$ & 0.431 \\
\hline BMI & $27.95(4.85)$ & $28.22(4.97)$ & $0.27(0.08,0.45)$ & $26.91(3.36)$ & $26.98(3.41)$ & $0.08(-0.38,0.53)$ & $0.72(59)$ & 0.401 \\
\hline SBP & 131.24 (15.66) & $132.52(15.96)$ & $1.27(-3.23,5.77)$ & $138.50(12.93)$ & $131.21(14.15)^{*}$ & $(-13.21$ & $5.71(59)$ & 0.02 \\
\hline DBP & $78.42(10.37)$ & $81.70(9.43)$ & $3.27(0.24,6.31)$ & 82.39 (7.66) & $77.61(9.48)^{\star \star}$ & $-4.79(-8.15,1.42)$ & $13.26(59)$ & 0.001 \\
\hline
\end{tabular}

${ }^{*} \mathrm{P}$-value of within group statistics was $0.018 ;{ }^{* *} \mathrm{P}$-value of within group statistics was 0.007 .

\section{Discussion}

In this study, instant brown rice powder was shown to be promising in reducing $\mathrm{HbA} 1 \mathrm{c}$, although the reduction was not significant. The improvement in HbA1c levels in the BR group may be explained by the fact that brown rice generally has a low glycemic index and lower area under the glucose curve, which produces low glycemic responses $[15,16]$. Our results are similar to those of a previous study, which substituted a white rice diet with a brown rice diet [17]. The non-significant finding may have been due to the short-term duration of the study, participants' lower HbA1c levels upon recruitment, and the similar level of the glycemic index of the products given to both arms of the study. Another study demonstrating a significant reduction in $\mathrm{HbA} 1 \mathrm{c}$ used brown rice as part of a vegetarian diet. In that study, HbA1c levels upon recruitment were between $6.0 \%$ and $11.0 \%$, with a mean HbAlc level of $7.7 \% \pm 1.3[18]$. The difference between these results and those of the current study may be due to the former study's addition of brown rice as part of a vegetarian diet and the complete replacement of white rice with brown rice instead of taking the latter as a supplement, as in our study. Similarly, a non- significant trend in favor of the BR group was found for fasting blood sugar (FBS).

\section{Lipid outcomes}

The results in this study showed a significant reduction in total cholesterol and triglyceride levels. Various studies have shown the benefits of rice bran in lowering cholesterol levels [5,6]. Gamma-oryzanol, which is in rice bran, interferes with the intestinal absorption of cholesterol in the diet [19]. In vitro studies have also shown that the contents of rice bran are capable of binding to bile acids and reducing the activity of HMG-CoA reductase, the key enzyme in the endogenous pathway of cholesterol metabolism [20].

Another possible mechanism by which brown rice could affect cholesterol in the body is through its fiber content. A metaanalysis completed in 1999 found that soluble fiber was able to reduce total cholesterol and LDL cholesterol levels [7]. There is a dose-response relationship between the amount of soluble fiber and the reduction in total cholesterol and LDL cholesterol levels. The contents of dietary fiber in our investigational product were $1 \mathrm{~g}$ per serving. Thus, with three servings per 
day, the participants in the intervention group should have received an additional $3 \mathrm{~g}$ of dietary fiber in their daily diet.

In our study, as the investigational product was pure brown rice powder, it shared similar biochemical and nutritional properties of rice bran in brown rice-thus the notable improvements in triglycerides and total cholesterol. However, the failure to yield any difference in HDL and LDL cholesterol levels may be attributable to the use of the investigational product as a supplement as well as to the short study duration.

Surprisingly, there is a significant reduction in the blood pressure in the BR group. This reduction in blood pressure in the brown rice group was also demonstrated in a number of other studies [8,9]. These blood pressure effects may be attributable to the higher fiber content and multiple micronutrients and vitamins contained in brown rice [21].

\section{Conclusion}

Instant brown rice as a dietary supplement in patients with type 2 diabetes shows promising beneficial effects, especially for improving total cholesterol, triglyceride levels, and blood pressure. However, further studies with larger sample sizes and longer durations are needed to further evaluate the long-term effects of brown rice as a dietary supplement among diabetic patients.

\section{Acknowledgement}

We would like to thank Prof. Dr. Mafauzy Mohammed and Mustapha Umar Imam who have contributed to this research. At the same time, we sincerely thank those who participated in the study.

\section{References}

1. Organization WH. Global report on diabetes. France: World Health Organization 2016.

2. Mathers CD, Loncar D. Projections of global mortality and burden of disease from 2002 to 2030. PLoS Med 2006; 3: 442.

3. Kamaruddin NA, Omar AM, Muhayidin AD, Yusof BNM, Pheng CS, Swee WCS. Management of type 2 diabetes mellitus. Putrajaya: Malaysian Endocrine and Metabolic Society Ministry of Health Malaysia 2015.

4. Morrison F, Shubina M, Turchin A. Lifestyle counseling in routine care and long-term glucose, blood pressure, and cholesterol control in patients with diabetes. Diab Care 2012; 35: 334

5. Evert AB, Boucher JL, Cypress M, Dunbar SA, Franz MJ, Mayer-Davis EJ. Nutrition therapy recommendations for the management of adults with diabetes. Diab Care 2013; 37: 120.

6. Franklin BA, Durstine JL, Roberts CK, Barnard RJ. Impact of diet and exercise on lipid management in the modern era. Best Pract Res Clin Endocrinol Metabol 2014; $28:$ 405-421.
7. Brown L, Rosner B, Willett WW, Sacks FM. Cholesterollowering effects of dietary fibre: a meta-analysis. Am J Clin Nutr 1999; 69: 30-42.

8. Bui TN, Le TH, Nguyen DH, Tran QB, Nguyen TL, Le DT. Pre-germinated brown rice reduced both blood glucose concentration and body weight in Vietnamese women with impaired glucose tolerance. J Nutr Sci Vitaminol 2014; 60: 183-187.

9. Wang B, Medapalli R, Xu J, Cai W, Chen X, He JC. Original article: Effects of a whole rice diet on metabolic parameters and inflammatory markers in prediabetes. eSPEN J 2013; 8: 15-20.

10. $\mathrm{Hu}$ EA, Pan A, Malik V, Sun Q. White rice consumption and risk of type 2 diabetes: meta-analysis and systematic review. BMJ 2012; 344: 1454.

11. Sun Q, Spiegelman D, van Dam RM, Holmes MD, Malik VS, Willett WC, Hu FB. White rice, brown rice, and risk of type 2 diabetes in US men and women. Arch Intern Med 2010; 170: 961-969.

12. Barclay AW, Petocz P, McMillan-Price J, Flood VM, Prvan T, Mitchell P. Glycemic index, glycemic load, and chronic disease risk-a metaanalysis of observational studies. Am J Clin Nutr 2008; 87: 627-637.

13. Hodge AM, English DR, ODea K, Giles GG. Glycemic index and dietary fiber and the risk of type 2 diabetes. Diabetes Care 2004; 27: 2701-2706.

14. Hayakawa T, Suzuki S, Kobayashi S, Fukutomi T, Ide M, Ohno T. Effect of pre-germinated brown rice on metabolism of glucose and lipid in patients with diabetes mellitus type 2. J Jap Assoc Rural Med (Japan) 2009; 58: 438-446.

15. Karupaiah T, Aik CK, Heen TC, Subramaniam S, Bhuiyan AR, Fasahat P. A transgressive brown rice mediates favourable glycaemic and insulin responses. J Sci Food Agr 2011; 91: 1951-1956.

16. Mohan V, Spiegelman D, Sudha V, Gayathri R, Hong B, Praseena K. Effect of brown rice, white rice, and brown rice with legumes on blood glucose and insulin responses in overweight Asian Indians: a randomized controlled trial. Diab Technol Ther 2014; 16: 317-325.

17. Zhang G, Pan A, Zong G, Yu Z, Wu H, Chen X, Tang L, Feng Y, Zhou H, Chen X, Li H, Hong B, Malik VS, Willett WC, Spiegelman D, Hu FB, Lin X. Substituting white rice with brown rice for 16 weeks does not substantially affect metabolic risk factors in middle-aged Chinese men and women with diabetes or a high risk for diabetes. J Nutr 2011; 141: 1685-1690.

18. Lee YM, Kim SA, Lee IK, Kim JG, Park KG, Jeong JY. Effect of a brown rice based vegan diet and conventional diabetic diet on glycemic control of patients with type 2 diabetes: a $12 \mathrm{w}$ randomized clinical trial. PLoS One 2016; 11: 1-14.

19. Cicero AFG, Parini A, Rosticci M. Nutraceuticals and cholesterol-lowering action. IJC Metabol Endocr 2015; 6: 1-4. 
20. Kahlon TS, Woodruff CL. In vitro binding of bile acids by rice bran, oat bran, barley and beta-Glucan enriched barley. Cereal Chem 2003; 80: 260-263.

21. Ascherio A, Rimm EB, Giovannucci EL, Colditz GA, Rosner B, Willett WC, Sacks F, Stampfer MJ. A prospective study of nutritional factors and hypertension among US men. Circulation 1992; 86: 1475-1484.

\section{*Correspondence to}

Lili Husniati Yaacob
Department of Family Medicine

School of Medical Sciences

Health Campus Universiti Sains Malaysia

Malaysia 